\section{DEL AISLACIONISMO A LAS ALIANZAS}

\author{
Francisco Michavila \\ Cátedra UNESCO de Gestión y Política Universitaria
de la Universidad Politécnica de Madrid \\ Cátedra UNESCO de Gestión y Política Universitaria
de la Universidad Politécnica de Madrid
}

\section{FROM ISOLATIONISM TO PARTNERSHIPS}

\begin{abstract}
The universities have passed to look within to seek opportunities for collaboration, either between universities or with public and private entities in other areas. The construction of the European Higher Education Area and the internationalization of universities have made cooperation and partnerships an emerging value for the future. In a scenario of competition between universities, partnerships are essential for the multiplication of opportunities in attracting top students, teachers and university researchers, and to improve the results of university activity.
\end{abstract}

KEY WORDS: Educational policies; university strategies; university cooperation; learning improvement.

\section{Parte del pasado, parte del futuro}

La Universidad ha tenido en el pasado una imagen distante. Para muchos ciudadanos, para casi todos, era una institución desconocida, de cuya actividad apenas se sabía, salvo que alli se podía acudir a clase y que, tras superar un importante número de exámenes, se podía obtener un título. Sólo "los iniciados" explicaban que los profesores se dedicaban -eso sí: solo una minoría- a otras cosas distintas que a dar clase. Investigan, decian, como quien alude a algo raro o inhabitual.

Si la sociedad era desconocedora de muchas de las actividades de los universitarios, a éstos poco les importaba que fuese así. Se decía que la Universidad era una especie de "torre de marfil", que estaba aislada y que era insensible a las expectativas de progreso social de los ciudadanos. Sus puertas se abrían de la misma manera cada día, con independencia de lo que ocurriese fuera de ella. Si los jóvenes se matriculaban en mayor o menor medida en una carrera determinada poco importaba. Si aprobaban solo unos pocos alumnos ciertas asignaturas,
RESUMEN: Las universidades han pasado de mirar al interior a la búsqueda de oportunidades para la colaboración, sea entre universidades o con entidades públicas y privadas de otros ámbitos. La construcción del Espacio Europeo de Educación Superior y la internacionalización de las universidades han convertido a la cooperación y las alianzas en un valor emergente para el futuro. En un escenario de competencia entre universidades, las alianzas son indispensables para la multiplicación de oportunidades en la captación de los mejores estudiantes, profesores e investigadores universitarios, así como para favorecer los resultados de la actividad universitarias.

PALABRAS CLAVE: Políticas educativas; estrategia universitaria; cooperación universitaria; mejora del aprendizaje.

casi nada importaba. Si algunos jóvenes, o muchos, se atascaban en alguna asignatura de sus estudios, año tras año a base de suspensos, y abandonaban a la postre, poco importaba. Incluso nadie, o muy pocos, respondían del despilfarro de ilusiones o de dinero que representaba semejante actitud.

La idea de la rendición de cuentas a la sociedad, de explicar qué hacen los departamentos universitarios y cómo lo hacen es reciente, muy reciente, tiene menos de tres decenios de vida. Más aún, el profesor que era excesivamente riguroso a la hora de aprobar a sus estudiantes incluso gozaba de reputación o un cierto reconocimiento social. Es un hueso, decían, lo que significaba una forma de reputación. La Universidad no explicaba lo que hacia, y a nadie le importaba que esto fuese así.

El prestigio de la enseñanza universitaria con frecuencia ha estado, y aún lo está, en determinados ámbitos relacionado con el carácter selectivo de los programas de estudio. La dificultad del aprobado algunos la confunden con la excelencia de un tipo determinado de estudios. Si suspenden 
muchos alumnos, algunos lo interpretan como un indicador de calidad y confunden la selección con la formación; no importa si lo que han aprendido es útil para algo y si, con el paso de los años, va a servir para su formación como profesionales y como ciudadanos.

Lo único que se destacaba, o se destaca todavía hoy en bastantes ocasiones, es si el estudiante ha superado la criba que constituyen unos exámenes difíciles y es "de los mejores". Pero, ¿los mejores, en qué? ¡Cuántas energías juveniles malgastadas en empresas estériles! ¿Quién no ha oído hablar de lo difícil que era superar no hace demasiados años las pruebas del ingreso en las escuelas de ingeniería? Algunos incluso lo añoran.

No en todas las universidades, ni en todos los países, ni en todos los tiempos, han sido así las cosas. La universidad española en los oscuros tiempos del franquismo era vista principalmente, por los que mandaban y marcaban las pautas del país, como una institución que convenía que estuviese lo más adormecida posible, y que se limitase a una rutinaria concesión de diplomas con la que los jóvenes se incorporaban a la vida laboral. Les preocupaba que molestase lo menos posible. La investigación era poco reconocida, poco apoyada con recursos y considerada como una actividad más propia de personajes excéntricos. La vocación científica tenía en aquel tiempo una lectura casi mística. 0 sea, que los campus universitarios debían generar el menor ruido posible.

Cierto es que ellos tenían razón, o sus razones, en que era lo que convenía en la defensa de sus espurios intereses, como se pudo comprobar en los últimos años de la dictadura, puesto que las energías de los jóvenes universitarios y de los profesores más inquietos iban a orientarse a la oposición al régimen y la reconquista de la libertad robada durante casi cuatro decenios.

Por el contrario, en otros países o en otras épocas más felices, las universidades han sido también las que han marcado las pautas culturales, los avances científicos y de progreso social. Han gozado de un prestigio inmenso y los ciudadanos se han sentido orgullosos de su existencia.

Las ciudades que han sido consideradas como ciudades universitarias lo han valorado como un plus respecto a las demás, como una seña de identidad que las situaba en una posición ventajosa ante otras poblaciones de tamaño semejante.

No puede entenderse ni concebirse el progreso de la sociedad norteamericana sin valorar la influencia decisiva que en él han tenido los jóvenes formados en Harvard, Beckley o Princeton desde hace más de un siglo. Francia sin la Sorbona no sería lo que es, tampoco la sociedad británica sin Oxford o Cambridge, o Alemania sin Heidelberg.

Las universidades prestigiosas han progresado habitualmente de modo independiente. Cada una lo ha hecho por su cuenta, de modo independiente, habitualmente. Si el aislacionismo de los centros españoles, cuando no una rivalidad exacerbada o frecuentemente sin sentido entre los profesionales en ellos formados, ha sido característico, tampoco la colaboración o las alianzas estables ha sido el modo de trabajo corriente en las buenas instituciones de otros países.

Siempre se pueden citar casos concretos que contraponer a ese individualismo, pero de un modo general cabe afirmar que la colaboración permanente o las alianzas en proyectos educativos o investigadores entre universidades más allá de las fronteras de los estados es un valor emergente del nuestros días. Si alguien pretendiera simplificar, por medio de un mensaje sencillo, la respuesta idónea sobre cuál es el camino que deben recorrer las instituciones académicas dedicadas a la educación superior y a la investigación en el próximo futuro -es decir, si pretendiese responder a la transcendente pregunta "¿dónde vas Universidad?"- esta podría ser: del aislacionismo a las alianzas. Las siguientes generaciones lo hallarán tan lógico y normal que pensarán que siempre fue de este modo.

¿Cómo construir el mañana de los universitarios?, ¿se trata de hacer todo de una forma diferente a como se ha hecho hasta ahora? Con lo nuevo y con lo antiguo, a la vez, en armonía. Cogiendo lo mejor de aquí y de allí, aprendiendo de lo bien hecho ayer y de lo que se desea mejorar hoy.

En un discurso que pronunció en París en 1994, con el título Las transformaciones sociales del siglo XX, Alain Touraine decía: "Ahora sabemos que siempre se construyen cosas nuevas con otras viejas, y que la modernidad no consiste en borrar el pasado sino en incorporar todo lo posible del pasado en todo lo posible del futuro". La visión de Toura- 
ine es también muy conveniente a propósito de lo que deben hacer las universidades europeas en los próximos años. No se trata de acabar con el pasado, como él dice con clarividencia, sino de impulsar la búsqueda de nuevos conocimientos y las formas renovadas que se deben utilizar en su transmisión, poniendo el acento en sus beneficiosos efectos en el progreso social.

Por resumir lo anterior de alguna manera, se podría, en definitiva, afirmar que la buena ruta que debe seguirse en la construcción del futuro los campus universitarios consiste en hacer mejor lo que ya se hace bien y hacer bien otras cosas nuevas. Esto no debe resultar extraño o ajeno a los integrantes de la academia que siempre se ha caracterizado por su elevado sentido de la autocrítica.

\section{LOS BUENOS EJEMPLOS}

De la historia particular de cada una de las universidades o de la evolución, en una visión más moderna o más reciente de la cuestión, de los sistemas universitarios entendida de modo global, se pueden extraer muy buenas lecciones para construir su porvenir. Algunos dicen, y no les falta razón, que el programa Erasmus recupera el espíritu que alimentaba a los estudiantes universitarios medievales, cuando iban de una ciudad a otra en busca del maestro deseado. La búsqueda del conocimiento no puede detenerse en fronteras más o menos artificiales. La diferencia se halla ahora en el papel que juegan los gobiernos $y$, sobre todo, en el número de los jóvenes que viven esa experiencia vital.

La idea, en su esencia cualitativa, es antigua; sin embargo, la extensión de la misma a muchos más ciudadanos que se dedican a estudiar, sin la limitación de que el patrimonio de sus familias lo permita o no, es nueva. Decía Touraine en la citada alocución que París (Touraine, 1994) se modernizó en las dos últimas décadas del siglo pasado creando un conjunto de grandes museos. Lo cual tiene algo de paradoja unamuniana.

Analicemos la idea anterior bajo el prisma de un ejemplo concreto. La historia del Massachusetts Institute of Technology, conocido popularmente por sus siglas: el MIT. De su estudio se pueden extraer interesantes conclusiones sobre cómo proyectar con sabiduría lo mejor del tiempo pasado en el futuro. Hace 150 años que se fundó. Lo hizo el geólogo William Barton Rogers en 1861, y lo sustentó sobre cuatro principios esenciales: el valor educativo de la aplicación de los conocimientos, la responsabilidad social, el aprendizaje activo y la combinación de la educación profesional con la educación liberal.

Eso se dijo hace nada menos que siglo y medio, pero si me perdonase el lector un "pequeño juego" con la cita y en este texto mencionase esas ideas no como son, sino como extraídas de alguna de las comunicaciones recientes de la Comisión Europea, a propósito de la modernización de las universidades de nuestro continente y las expectativas o esperanzas que hay en ellas depositadas, no creo que fuese demasiado atrevido afirmar que tal argucia generaría ciertas dudas en el lector. Bastantes las calificarían de rompedoras.

Sigamos con el ejemplo. Mente y manos, es el lema del MIT. Algo que, por otra parte, resultará familiar y próximo a quienes sean conocedores de la visión y los fundamentos del más importante proyecto educativo que se ha dado en la historia de la educación en España, la Institución Libre de Enseñanza. El sueño de Giner, continuado por Cossío y plasmado en acción transformadora por Castillejo. Mente y manos ayer, mente y manos hoy, mente y manos en una de las más prestigiosas instituciones americanas, que cuenta con cerca de 80 Premios Nobel entre sus antiguos alumnos y docentes, mente y manos en la Institución Libre de Enseñanza, cuyas ideas alentaron y sustentaron el mayor proyecto de renovación social y modernidad en España, que pretendió hacer realidad la República de Azaña.

Ayer y hoy. Si ayer, en su fundación, el MIT proponía una visión de la educación universitaria avanzada a su tiempo, hoy también lo hace con un verbo renovado. Susan Hockfield, su actual presidenta, insiste en sus discursos en la voluntad pionera de la institución. Cuando reflexiona en algún texto de presentación de sus perspectivas académicas (Hockfield, 2011), sobre cómo el MIT puede ayudar a construir un mundo mejor, Hockfield alude a sus orígenes e insiste en la aplicación de las capacidades adquiridas, por medio de los estudios allí cursados, y pone un énfasis singular en la capacidad para aplicarlos a problemas interdisciplinarios. Destaca igualmente la importancia que conceden en el MIT a temas y asuntos críticos en el deve- 
nir del mundo actual: el cambio climático, la pobreza, el futuro de nuestras ciudades, etcétera.

También, la Task Force on Student Life and Learning creada hace pocos años por el MIT ha añadido otros principios formativos emergentes a los que en su día estableció Rogers: una visión integrada de los valores académicos, la investigación y de la sociedad, la diversidad como valor transversal y que la intensidad, la curiosidad y el entusiasmo sean las características principales de la formación adquirida.

No es ni mucho menos el MIT el único ejemplo al que acudir para adentrarse en la aventura de cómo combinar lo nuevo con lo antiguo. Se podrían citar bastantes ejemplos en ambas orillas del Atlántico. Una brillante idea aquí, un proyecto visionario allá. Sin embargo, son impulsos aislados casi todos, pocos esfuerzos colectivos, nacionales 0 continentales. Quizá una de las excepciones más notables lo constituya la creación del Espacio Europeo de Educación Superior, en el que lo más singular que tiene es precisamente ese valor añadido.

En este texto no se pretende realizar ningún recorrido por la historia de las universidades. Hay tratados que lo hacen con solvencia. Baste solo añadir una apostilla al somero relato de la buena lección que puede aprenderse del prestigioso Instituto que se emplaza en la ciudad de Boston. Se refiere al sentido casi eterno, o si se prefiere atemporal, que impregna al buen hacer de las más prestigiosas universidades del mundo. Supera su tiempo y su localización geográfica. Con una imagen tan solo basta para simbolizarlo: la grandeza, la solemnidad y el respeto por la ciencia están sintetizadas en la monumentalidad del edificio central de la Sorbona, reconstruido por la Tercera República francesa y en cuyas paredes se ven retratados bastantes de sus sabios profesores, unos de tiempos más lejanos y otros más cercanos a nuestros días. ¿Quién no siente una emoción especial cuando cruza su puerta y deja a la espalda la estatua de Auguste Compte, en la pequeña plaza por la que se accede? En las raras veces que se abre su puerta principal, el espectador que lo presencia siente que algo especial celebra la humanidad, y que aquella entrada se da la bienvenida a la sabiduría.

La cuestión no es sencilla. La piedra angular del proyecto universitario global sobre la que se sustente el proceso de armonización de la educación superior de los europeos consistente en cómo en Europa se puede pasar de un conjunto de universidades, algunas muy buenas y otras muchas de nivel inferior, a un sistema universitario europeo, armónico y coordinado. Un espacio común donde, por afinidad científica o por objetivos académicos, trabajen juntos en proyectos compartidos grupos o equipos de profesores de ámbitos culturales, lingüísticos o geográficos diferentes. No se trata de sustituir los pequeños grupos tradicionales por otros mayores juntándolos todos en un mismo lugar.

La cuestión se refiere a que en el tiempo actual, con el uso de las extraordinarias capacidades de comunicación que se disponen, se sumen los esfuerzos de aquéllos que estén interesados en generar sinergias. No se trata de reducir la excelencia de nadie sino de extender los conocimientos y sus beneficios a cuantos más pueblos o colectividades sean posibles. La palabra clave no es fusión, es alianza. No consiste en grandes conglomerados de profesores y estudiantes. La suma de las capacidades diversas de actores plurales, en pos de la excelencia académica. La alianza de los que son complementarios, no clónicos. La diversidad entendida como riqueza, en un contexto de coincidencia de los intereses científicos de unos y otros.

Llagados a este punto, quizá sea oportuno destacar que un defecto bastante extendido entre los políticos cuando alcanzan el poder es creer que hay que cambiarlo todo, que lo anterior poco vale y que el que llega lo hace con la pócima mágica. Con frecuencia les falta la grandeza de espíritu suficiente para no creerse poseedores de la verdad revelada, de modo nada de lo que hicieron sus antecesores es aprovechable. 0 sea, han de inventarlo todo, como es un hábito extendido entre los llamados nuevos ricos, para quienes lo que interesa es lo superficial, las apariencias, o que sirve de justificación de los que hacen de la tecnocracia su profesión. Aquí, como indica el título que antecede a estas páginas, el pasado se reinterpreta para que se pueda aprovechar su posible contribución en la construcción de un futuro fértil para los campus universitarios.

La armonía entre la competencia y la colaboración es la esencia de casi todo. Tan difícil como necesaria. Pensar, con palabras y pensamientos actuales, el mañana de la educación superior y la investigación científica es una tarea que ha de realizarse con altas dosis de humildad. Esa 
humildad que es la virtud esencial de todo investigador. Nada indica que lo que proceda acometer sea una ruptura "iluminada". Pero sí que hay que tener creencias firmes. Una de ellas es que la renovación, que por otra parte ha de ser un hecho permanente porque así lo exige la propia esencia universitaria, del modo en que se trabaja en los campus aumentará su sensibilidad ante las crecientes expectativas ciudadanas y su compromiso con la solución de los problemas sociales.

De las universidades europeas, y en particular de las españolas, se espera mucho. No ya que instruyan a los jóvenes en una profesión o que, siendo más ambiciosos, los eduquen como ciudadanos activos, sino también se esperan contribuciones científicas notables al progreso tecnológico y social de los pueblos.

La docencia y la investigación no son separables a la hora de concebir el futuro universitario. Si durante un tiempo algunos hablaron de la especialización de los campus de manera que se sugería la posibilidad de que hubiese universidades dedicadas solo a la docencia, ahora ya no hay una corriente solida de opinión que apoye la idea. Esa visión ha quedado obsoleta. Los asuntos de auténtico calado, los que en realidad interesan y sirven a los interese colectivos, son otros, como no es difícil entender. Las universidades tienen que ser plurales, cuanto más diferentes sean mejor, pero el principio que sustenta su existencia se basa en la síntesis y la indisolubilidad entre las actividades educativas y las tareas de investigación. Si se abordan por separado, si se rompe el binomio, se pierde la esencia de lo que debe ser el élan vital de los centros de educación superior e investigación.

En contraposición con lo que ocurría en su tiempo en Francia, con el denominado modelo napoleónico, Wilhelm Von Humboldt, formado en la reputada ya por entonces Universidad de Götinga, junto a su hermano Alexander, y cuyo apellido se incorporó en 1949 al nombre previo para dar paso al actual de la Universidad de Berlín, fue quien propugnó que la universidad fuese así. Humboltd sintetizaba las reflexiones previas de pensadores alemanes como Fitche o Hegel.

Educación y ciencia, a la vez. Todo ello amparado por las ideas de la libertad de la ciencia y la autonomía académica. La autonomía universitaria está recogida en la actual
Constitución española, y sobre ella tanto se ha escrito y hablado en los tiempos más recientes. ¡Esa autonomía por la que se suscitaron tantas disputas en nuestros centros en los primeros cursos del actual siglo! Aquel modelo alemán es ahora aceptado como el modelo europeo de universidad. En él se funda, y se fundará con más vigor cuando deje de azotar la sociedad el vendaval contrario generado por la crisis económica, la culminación de la construcción del área europea de la educación superior y la investigación. Además, en cada región geográfica y en cada disciplina académica los campus se deberán especializar, de manera que sean complementarios en su conjunto.

Si el principio de educación para la ciencia es el punto de partida, las reformas educativas constituyen otro pilar esencial para sustentar las innovaciones académicas. Tampoco es éste un asunto totalmente original de nuestra época. No será el primer intento, ni lo ha sido de los que se han ocupado de la renovación de las metodologías educativas ni tampoco de aquellos otros tendentes a innovar en los sistemas de evaluación de los saberes adquiridos por los estudiantes. Ambos son solo dos, pero de los más destacados, de los asuntos cruciales para avanzar en el camino que lleva a la sustitución de la enseñanza tradicional por una educación activa.

Son bastantes los precedentes que existen en cuanto a innovaciones en las formas de enseñar y educar a los universitarios; los hay de diversos intentos de introducir mejoras, y también de esfuerzos baldíos en casi todas las ocasiones. Las buenas prácticas que pueden mencionarse no se encuentran en España, al menos en una escala apreciable. Unos son más utópicos, otros más propios de la acción reformadora llevada a cabo por académicos o gobernantes moderados.

Permítame el lector acudir a otro ejemplo. La referencia hace mención de la voluntad renovadora de un gobierno en cuanto a la organización de los procesos de transmisión de conocimientos. Tuvo lugar en España, en los años treinta, en el bienio de gobierno de la denominada conjunción republicano-socialista. La iniciativa educativa la recogió el proyecto de ley de la reorganización de la ordenación de los estudios en las universidades, presentado por el ministro de Instrucción pública y Bellas Artes, el socialista Fernando de los Ríos en marzo de 1933 para su tramitación parlamentaria. En el Preámbulo que antecedía al 
articulado de aquel texto legislativo, que desarrollaba una ley non nata porque no fue aprobada a causa de la disolución anticipada de las Cortes, se recogían pensamientos, comparaciones entre sistemas de enseñanza superior de diversos países y diversos análisis sobre el mejor encaje que era posible entre la educación superior y la ciencia. Se atrevía, incluso, el borrador presentado a tratar la manera de reformar el sistema de exámenes vigente entonces en la educación superior española. Recogía las ideas de Giner, a quien citaba expresamente, de que el examen altera en su esencia la enseñanza "porque no se estudia para saber y por saber, sino para examinarse. De aquí que la idea del examen con preocupación deba de desaparecer de la mente de los alumnos".

Si este pensamiento, y su plasmación en un texto de carácter legal, puede calificarse sin exageración de avanzado o atrevido, no lo es menos la forma en que se abordan otros asuntos en aquella reforma universitaria. La conexión entre la universidad y las aspiraciones sociales que debe atender o el papel principal que daba a la investigación y los estudios de doctorado, son otras pruebas notables de las buenas cualidades que la amparaban.

La posterior Ley de Reforma Universitaria de 1983 hereda y atempera varios de los principios allí establecidos. No cabe duda de que el factor cuantitativo hacía difícil, cincuenta años después, la aplicación alguna de las nobles intenciones esbozadas en la norma elaborada por Fernando de los Ríos y sus colaboradores. Pero nadie puede negarle su atrevimiento y sólida fundamentación pedagógica.

Gobernantes y académicos. Si la cita anterior corresponde a una voluntad reformadora alentada desde el poder político, hay otros casos también notables de cómo las mismas instituciones pueden preocuparse por la idónea orientación que deben dar a sus tareas, y adaptar sus objetivos docentes e investigadores a las demandas de la comunidad en la que nacen. Una experiencia destacada al respecto se refiere a los motivos que alentaron el nacimiento de la Penn State University y su posterior evolución (Penn, 2011). Su ubicación en State College, en el corazón de Pennsylvania, alejada de Philadelphia y de Pittsburg, situada como se sitúa el punto de corte -algunos afirman que en el centro de la nada en aquel tiempo remoto- de las dos diagonales de un rectángulo, que es la forma geométrica de ese Estado, y su orientación primera hacia la agricultura contribuyeron mucho al progreso de ese territorio americano.

Fue Evan Pugh quién en 1862, cuando el campus apenas tenía siete años de vida, supo ir más lejos de sus primeras intenciones agrícolas y encontrar un equilibrio entre los estudios clásicos y las aplicaciones prácticas. Las innovaciones que introdujo iban encaminadas a formar a los ciudadanos de la joven república norteamericana en las materias que más se demandaban o convenían para su desarrollo. En la aclimatación a las nuevas tierras, la agronomía tenía un lugar prominente. En este caso se podría decir que las ideas originales surgieron desde la base, desde aquel primer grupo de profesores encabezados por Pugh.

En el ejemplo previo otros profesores metidos a gobernantes, casi todos ellos institucionistas o seguidores de los pensamientos de Giner, fueron quienes desde el ejercicio de las responsabilidades políticas impulsaron renovaciones en los modos y maneras de educar. "Hombres nada revolucionarios, llenos de respeto, mesura y tolerancia" como los veía Antonio Machado.

El afecto se gana día a día. Si esto le ocurre a las personas, también es cierto para las organizaciones sociales. Por ese motivo, una universidad ha de esforzarse para hacerse querer. Más aún, para hacerse respetar. No se trata de nominalismos, de nombres que por ellos mismos generan adhesiones. El día a día, el trabajo constante, las puertas abiertas y la capacidad de escuchar a cuantos se acercan a una institución la hacen ser querida. Los ciudadanos se identifican con facilidad con las instituciones u organizaciones sociales si las perciben como modélicas $y$, a su vez, austeras.

Recuerdo mi propia experiencia en los apasionantes años que viví con motivo de la creación de la Universitat Jaume I de Castelló. Han transcurrido veinte años y los habitantes de su entorno ahora la valoran mucho, están orgullosos de ella. La propia universidad lo sabe. Le beneficia que sea así. Pero esto no ha surgido de la nada, no se trata de un movimiento espontáneo, es consecuencia de un esfuerzo constante y tenaz, de explicar lo que es y lo que aspira a ser. Buena dosis de comunicación y una sensibilidad especial para acoger a todos los que se aproximan con una sugerencia o una colaboración. En los años que pasé dirigiéndola, incluso en los meses previos que se dedicaron 
a los trabajos de diseño del proyecto que sustentó su nacimiento, una de las mayores prioridades que nos animaron en aquella noble tarea fue darla a conocer.

La meta era conseguir que cada uno de los ciudadanos, no únicamente los posibles alumnos de los cursos venideros, de toda el área metropolitana que la envuelve, la sintiesen como suya. Conseguirlo algo similar es una obra basada en la constancia, no es una declaración o una flor de un día. Día a día, paso a paso, es como han labrado su reputación los prestigiosos centros norteamericanos. Los resultados inmediatos en materia educativa son efímeros. La propaganda superficial, el marketing de corto vuelo vale poco para convencer o atraer en asuntos académicos.

La credibilidad de los campus universitarios o la solidez de su obra, tiene mucho que ver con dos cuestiones concretas: una se refiere al rigor del trabajo cotidiano con el que el centro universitario aspira a conseguir la excelencia académica, otra tiene que ver con la apertura a las demandas sociales. Ninguna aspiración puede estar excluida pero unas son más prioritarias que otras.

Las mejores universidades norteamericanas se fundamentan en la buena armonía y crecimiento de esa dualidad. En la hora actual, el liderazgo en los procesos de alianzas entre universidades está en sintonía con ese armónico crecimiento. Trabajo, mucho trabajo, y no alejarse de la senda por la que se encauzan las expectativas sociales. Tener voz autorizada y hacerla oír ante cualquier problema en su entorno, es de lo que se trata. ¿Qué otras organizaciones sociales gozan de tamaño privilegio?

Alguien podrá objetar que los cambios que se propugnan o los vientos renovadores que se anuncian tienen poco de concretos y mucho de retóricos. No es así. En absoluto, no es así. Aquí cabe el símil de que quedarse parado es retroceder. Universidad quiere decir progreso. Y atrevimiento. E inconformismo.

Veamos con un poco de detalle la cuestión. Situémonos en España. Una buena universidad española, por ejemplo una que esté situada en el primer tercio en su valoración por parte de alguna de las clasificaciones que hacen las agencias de calidad o los medios de comunicación, podría sentir la tentación de no introducir cambios, ahora, a causa de que las circunstancias sociales y económicas no son propicias. Esto es, huir de las grandes transformaciones en cuanto a métodos educativos o alianzas con otros campus de afinidades temáticas o ser selectiva en campos de investigación emergentes por considerarlos innecesarios en su caso. Acaso, argumentan, sería mejor esperar a que las condiciones las hagan más fáciles.

Si su labor está reconocida, si hablan bien de lo que ya vienen haciendo, ¿para qué introducir grandes alteraciones en su proceder? Podrían considerarlas, no es, acaso, como un riesgo innecesario. Esa actitud que a bote pronto puede considerarse prudente, incluso sensata, sería suicida a largo plazo pues sí que es necesario que las universidades se alejen de cualquier conformismo, por muchas complacencias que reciba de su entorno más local. Si no lo hacen, si permanecen aisladas, encerradas en sus rutinas docentes o investigadoras de corto vuelo, al cabo de unos años habrán visto pasar por delante las oportunidades sin aprovecharlas. Si las universidades son públicas, se deben además a su responsabilidad ante los ciudadanos que las sustentan con sus impuestos.

Acudamos a un tercer ejemplo, para clarificar esta última idea. La universidad Politécnica de Madrid de la que soy profesor está considerada como una de las buenas universidades españolas. La sociedad lo reconoce y valora la formación de sus titulados. Su participación en proyectos europeos es conocida y reconocida. Si sus aspiraciones para los tiempos venideros no fuesen más allá que las de ocupar un lugar confortable entre las universidades de su entorno, bien podría seguir durante bastantes años como hasta ahora, sin incorporar innovaciones fundamentales en su organización o sus metodologías educativas. Cuando su rector me encargó la dirección de la elaboración de su modelo educativo (UPM, 2011), encontré que ésta era la principal razón que podía esgrimir para pedir a su comunidad universitaria el esfuerzo gigantesco que significaba su adopción.

Pero hoy en día la medición de la excelencia académica se hace mediante comparaciones a escala internacional, como mínimo en el conjunto de Europa. La dimensión española es insuficiente, es demasiado local. La estrategia de incorporación al área europea de educación superior e investigación ha de consistir para la Universidad Politécnica de Madrid, como pasa para otras universidades españolas semejantes, en renovarse y en organizarse mejor, para ser 
competente, y de este modo que pueda competir con las mejores instituciones de tecnología de otros países. Si su aspiración es ésta, no le basta con seguir hacia adelante por inercia. Si lo hiciese al cabo de no demasiados años los daños serían irreparables, y la universidad quedaría en una situación de irrelevancia.

\section{El beneficio de las alianzas}

Las universidades que permanezcan aisladas carecerán de futuro. Las universidades no pueden aspirar a comportarse como meras academias dedicadas a la preparación de los jóvenes para una profesión. En ese tipo de tareas se verían superadas por otras organizaciones especializadas al respecto y que no necesitan del tipo de instalaciones como los que poseen los campus universitarios. Participar en las redes europeas dentro de poco no será una opción sino una obligación.

Tendrá la universidad que renovar sus objetivos y sus programas de trabajo, en tareas docentes y en actividades investigadoras. Las alianzas con otras instituciones en el marco de proyectos de investigación compartidos, la elaboración de una oferta de másteres capaces de ser atractivos de manera internacional o la incorporación de un modelo educativo que plasme sus virtudes formativas son cambios imprescindibles para situarse en el nuevo y más amplio escenario europeo.

Competencia y cooperación son dos principios activos en el porvenir de las universidades. En el pasado reciente se ha destacado fundamentalmente la competencia. Pero hoy no se conciben uno sin el otro. Participar en convocatorias competitivas, ser mejores que otros, destacar por encima de los demás, adelantar a la universidad vecina en cuanto al número de alumnos son varias de las características que se han destacado en los últimos años como síntomas de modernidad según algunos. Constituían la tarjeta de presentación en cualquier foro al que acudiesen. Pero la competencia sin más no es una buena fórmula. Incluso puede traer perjuicios a la comunidad si se imponen criterios utilitaristas, destinados a superar a los demás como fin último. La colaboración, y la cooperación si se quiere decir así, constituyen en contrapunto de la competencia. La búsqueda permanente del equilibrio entre ambas define el buen hacer. Esta afirmación es otra forma de abundar en la conveniencia de caminar por la senda que lleva de la mano el tránsito del aislacionismo a las alianzas. Un camino por el que se pueden a vislumbrar las universidades europeas un panorama optimista de futuro, y no otro de inspiración de rendimiento mercantil o de inspiración neoliberal.

Otra lección del pasado es que las grandes universidades, las que se aproximan o superan los cien mil estudiantes, no se corresponden con una adecuada organización de los recursos docentes e investigadores (Michavila, 2011). Si tiempo atrás se decía -creo recordar que en algún documento europeo se insiste en el tema- que el tamaño óptimo de las universidades se situaba entre veinte mil y cuarenta mil estudiantes, la consulta de las más prestigiosas clasificaciones internacionales o rankings universitarios, ratifica la bondad de esa estimación.

Las buenas universidades sajonas, norteamericanas o británicas, y las mejores de la Europa continental son de tamaño parecido entre sí. Unas apenas alcanzan los veinte mil alumnos, otras superan por poco los treinta mil, pocas de las ilustres Ilegan a los cuarenta mil. Así ocurre en Harvard, Stamford, Beckley, Yale, Oxford, Cambridge, Delft, Berlín, Oslo, Columbia, École Polytechnique Fédérale de Lausanne, Duke, etcétera. Por el contrario, su actividad no se acaba en el borde de sus campus. No hay fronteras entre ellas. Los profesores se desplazan con frecuencia de unas a otras. Los estudiantes cursan lo más atractivo de los programas con mayor movilidad que nunca. Las alianzas entre ellas son numerosas, por medio de planes conjuntos de investigación o de ofertas de programas de estudios de posgrado comunes, y crecen de manera exponencial con el tiempo.

Si se hace el ejercicio de pasearse por los jardines, los patios o los pasillos de los edificios del importante centro de investigación tecnológica que es la École ubicada en Lausanne, resulta difícil resistirse a la tentación de curiosear un poco sobre los nombres de los laboratorios que allí existen o sobre lo que dicen los paneles informativos. Quien lo haga se encontrará con el hecho de que muchos, no sé si la mayoría, son laboratorios mixtos con universidades que no son suizas, a menudo suelen ser norteamericanas 0 asiáticas. $Y$ este hecho es cada vez menos singular. 
Cuando alguien navega por internet buscando información sobre qué tipo de investigación hacen, y cómo la organizan, las universidades más prestigiosas de América y Europa, lo que en primer lugar destaca es que no actúan en solitario.
Las colaboraciones y las alianzas resaltan inmediatamente. Esta es una de las características indiscutibles del tiempo actual y lo será en un grado superior en los años que se avecinan para las universidades.

\section{BIBLIOGRAFÍA}

Hockfield, S. (2011): http://web.mit.edu/ hockfield/. Diciembre.

Michavila, F. (2011): "Alianzas, que no fusiones", El País, 15 de octubre.
Penn State University: http://www.psu.edu/ ur/about/mission.html.

Touraine, A. (1994): Las transformaciones sociales del siglo XX, Paris.

UPM (2011): http://www.upm.es/institucional. 